\title{
Therapiekosten unter der Lupe
}

- Glukokortikoidhaltige Nasensprays sind aus der symptomatischen Therapie der allergischen Rhinitis nicht mehr wegzudenken. Für viele HNO-Ärzte und Allergologen ist dabei Mometasonfuroat der Wirkstoff der ersten Wahl. Laut ArzneiverordnungsReport 2009 wird jedoch die Wirtschaftlichkeit des zugehörigen Präparats Nasonex ${ }^{\circledR}$ infrage gestellt, so dass verschiedene KVen es auf Me-Too-Listen führen. Daher kann der Wirkstoff von Ärzten nicht mehr uneingeschränkt eingesetzt werden, erklärte IMS ${ }^{\circledR}$-Health-Experte Detlef SchröderBernhardi, Frankfurt/Main. Er kritisierte, dass die Preise in dem Report über die Defined Daily Doses (DDD) - hier 0,2 mg - verglichen wurden, wonach für Mometason mit $0,89 €$ höhere Tagestherapiekosten als für das Referenzprodukt Budesonid mit 0,37 Euro resultierten. Diese angenommene mittlere Tagesdosis sei jedoch nur eine Ori- entierungsgröße, die nicht notwendigerweise der therapeutischen Äquivalenzdosis entspreche. Nur die konkret verordnete Dosis - auch als Prescribed Daily Dose (PDD) bezeichnet -, die Einflussfaktoren wie Schweregrad, Krankheitsdauer, Multimorbidität und Begleittherapien berücksichtigt, liefere die Grundlage für reale Tagestherapiekosten.

Eine Analyse durch IMS ${ }^{\circledR}$-Health sollte deshalb den tatsächlichen Verbrauch der beiden Wirkstoffe im Praxisalltag unter die Lupe nehmen. Hierzu wurden die Daten von Patienten, die Mometason bzw. Budesonid verwendeten, aus allgemeinärztlichen und HNO-ärztlichen Praxen für den Zeitraum von Oktober 2004 bis September 2008 ausgewertet; eine Subanalyse erfasste speziell Patienten mit allergischer Rhinitis.

Wie sich zeigte, benötigten die Patienten der Mometason-Gruppe im Vergleich zur
Budesonid-Gruppe diagnoseunabhängig nur halb so viele Sprühstöße und signifikant weniger Folgeverordnungen. Die Patienten, die ihre allergische Rhinitis mit Mometason behandelten, hatten einen niedrigeren adjustierten Pro-Kopf-Verbrauch (bis zu 360 Hübe weniger in 730 Tagen HNO-ärztlicher Therapie) und verursachten so merklich geringere Kosten als die entsprechende Budesonid-Gruppe. Summa summarum, folgerte Schröder-Bernhardi, sei die Therapie mit Mometason sogar kostengünstiger, wenn man den tatsächlichen Verbrauch zugrunde legt.

mju

Pressekonferenz „Neue Studie zur Wirtschaftlichkeit von intranasalen Kortikoidsprays“, im Rahmen des 4. Allergo-Rhinologischen Updates. München, 12. März 2010. Veranstalter: Essex Pharma, München

\section{Daten zu Rupatadin bestätigen Wirksamkeit und Sicherheit}

- Rund ein Jahr nach seiner Markteinführung hat sich der Wirkstoff Rupatadin (Rupafin ${ }^{\circledR}$ ) unter den Antihistaminika etabliert. Zwei aktuelle Studien bestätigen jetzt, dass der Wirkstoff, der zusätzlich zum $\mathrm{H}_{1}$-Rezeptor auch den PAF-Rezeptor blockiert, die Lebensqualität von Allergiepatienten verbessert und keine kardialen Effekte aufweist.

In der Esprint-V-Studie wurden die Patienten nach der Intensität ihrer Beschwerden in drei Gruppen eingeteilt (leicht: 2,5\%; mäßig: 52,8\%; schwer: 56,1\%) und vier Wochen lang mit Rupafin ${ }^{\circledR}$ behandelt. Von den Patienten, die zu Beginn der Studie

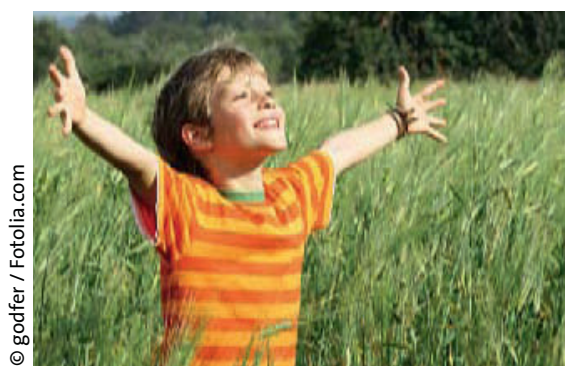

unter schweren Beschwerden gelitten, hatten, zeigten nach Therapieende mehr als die Hälfte $(56,1 \%)$ nur mehr leichte und 36,3\% mäßige Symptome. Aus der Gruppe der Patienten mit ursprünglich mäßigen Beschwerden konnten die Beschwerden bei über $60 \%$ auf ein leichtes Niveau reduziert werden. Anhand eines Fragebogen zeigte sich, dass Rupatidin darüber hinaus auch die Lebensqualität (z. B. Schlaf und psychische Belastung) verbessert.

In einer kürzlich veröffentlichten Studie [Donado E et al., Br J Clin Pharmacol 2010;(4):401-10] bestätigte sich auch erneut das gute Sicherheitsprofil des Wirkstoffs. Die randomisierte, doppelblinde und placebokontrollierte Studie zeigte, dass Rupatidin weder in der zugelassenen Dosierung von $10 \mathrm{mg}$ noch in einer zehnfach so hohen Dosis zu einer OT/OTCStreckenverlängerung führt und belegte damit erneut die kardiale Sicherheit des Antihistaminikums.

gz

Nach Informationen von Merckle Recordati

\section{Probiotika auf dem Prüfstand}

Eine Bestandsaufnahme der aktuellen Studienlage zu Probiotika stand im Mittelpunkt des von der Danone $\mathrm{GmbH}$ unterstützten Workshops „Probiotika evidenzbasiert - was ist wissenschaftlich erwiesen?" Neben Anwendungsgebieten wie Diarrhöen, Reizdarmsyndrom oder Nekrotisierender Enterokolitis bei Neugeborenen, bei denen die Datenlage bereits relativ gut ist, ging es bei dem Workshop auch um die Frage der Evidenz bei Allergien und Atemwegsinfektionen. Auch wenn in vielen Bereiche noch weitergehende Forschungsarbeiten nötig sind, gibt es doch bereits einige valide Daten wie z. B. hinsichtlich der Effekte bei Infektionen der Atemwege. Laut Prof. Jürgen Schrezenmeir, Karlsruhe, liegen mittlerweile Ergebnisse großer Studien mit insgesamt etwa 2.500 Personen vor, die zeigen, dass der Einsatz von Probiotika die Dauer oder die Inzidenz von Atemwegserkrankungen reduzieren kann.

\section{gz}

